\title{
REVISÃO DA EFICÁCIA DO USO DA LORCASSERINA PARA A PERDA DE PESO
}

\author{
EFFICIENCY REVIEW OF THE USE OF LORCASERIN FOR WEIGHT \\ LOSS
}

\author{
Luana Beatriz Camêlo ${ }^{1}$
}

Mielle Neiva Santos ${ }^{2}$

\begin{abstract}
RESUMO: Objetivo: Avaliar a eficácia da lorcasserina na perda de peso e observar outros possíveis benefícios. Método: Foi realizada uma pesquisa bibliográfica exploratória, com abordagem integrativa, com uma síntese qualitativa dos trabalhos encontrados. Resultados: Foram analisados 10 artigos nos quais evidenciou-se a eficácia da lorcasserina na diminuição do apetite e consequentemente no peso corporal, possivelmente devido a melanocortina 4 . O fármaco também auxilia na melhora da pressão arterial, frequência cardíaca, resistência à insulina e apneia do sono. Conclusão: A lorcasserina se mostrou eficiente quanto a redução de peso, melhoraria de vários fatores de risco e auxiliou no controle da fome, além de ter maior segurança cardiovascular e baixa incidência de efeitos adversos em relação a outros anorexígenos.
\end{abstract}

Palavras chave: Serotoninérgico. Obesidade. Perda de peso.

ABSTRACT: Objective: To evaluate the efficacy of lorcaserin in weight loss and look at other possible benefits. Method: An exploratory bibliographic research was conducted, with an integrative approach, with a qualitative synthesis of the works found. Results: We analyzed 10 articles in which the efficacy of lorcaserin in reducing appetite and consequently in body weight was evidenced, possibly due to melanocortin 4. The drug also aided in the improvement of blood pressure, heart rate, insulin resistance and sleep apnea. Conclusions: Lorcaserin was efficient in reducing weight, improving several risk factors and helped to control hunger, as well as having greater cardiovascular safety and low incidence of adverse effects in relation to other anorectics.

Keywords: Serotoninergic. Obesity. Weight loss.

\footnotetext{
${ }^{1}$ Graduanda do curso de Farmácia da Unifacisa Centro Universitário.

${ }^{2}$ Nutricionista e Professora do curso de Nutrição da Unifacisa Centro Universitário.
} 


\section{INTRODUÇÃO}

A obesidade é uma doença crônica não transmissível (DCNT) caracterizada pelo aumento excessivo da gordura corporal, geralmente associada ao superávit calórico, ocasionado por um consumo de calorias maior que o gasto energético. Atualmente, é um dos maiores problemas de saúde pública pois, além de ter aumentado muito nos últimos anos, é um fator de risco para diversas outras enfermidades (DIAS; HENRIQUES; ANJOS; BURLANDY, 2017; SOUZA et al., 2018).

O excedente calórico normalmente ocorre devido a uma alimentação desregular e/ou emexcesso em conjunto com o sedentarismo. Ademais, pode haver influência de fatores neurológicos, genéticos, endócrinos e sociais, que podem interferir no apetite, na facilidade de acumular gordura e no local onde ela é armazenada (CARVALHO; BELÉM; ODA, 2017).

De acordo com a Pesquisa de Vigilância de Fatores de Risco e Proteção para Doenças Crônicas por Inquérito Telefônico (VIGITEL) (BRASIL, 2019), o número de obesos no país aumentou $67,8 \%$ entre 2006 e 2018 e mais da metade da população, $55,7 \%$, tem excesso de peso, entre eles $19,8 \%$ apresentam algum grau de obesidade.

A Organização Mundial de Saúde (OMS) classifica a patologia com base no índice de massa corporal (IMC), que é determinado pelo cálculo do peso corporal, em quilogramas, dividido pelo quadrado da altura, em metros quadrados (IMC = $\mathrm{kg} / \mathrm{h}^{2}(\mathrm{~m})$ ), sendo o resultado desse cálculo usado para identificar se o indivíduo está com o peso ideal ou o classificar com sobrepeso ou algum grau de obesidade (Quadro 1). 
Quadro 1. Classificação do índice de massa corporal de acordo com a Organização Mundialde Saúde.

\begin{tabular}{|c|c|c|c|c|c|c|}
\hline Classificação & $\begin{array}{c}\text { Peso } \\
\text { Baixo }\end{array}$ & $\begin{array}{c}\text { Peso } \\
\text { Normal }\end{array}$ & Sobrepeso & $\begin{array}{c}\text { Obesidade } \\
\text { Grau I }\end{array}$ & $\begin{array}{c}\text { Obesidade } \\
\text { Grau II }\end{array}$ & $\begin{array}{c}\text { Obesidade } \\
\text { Grau III }\end{array}$ \\
\hline IMC $\left(\mathrm{kg} / \mathrm{m}^{2)}\right.$ & $<18,5$ & $18,5-24,9$ & $25-29,9$ & $30-34,9$ & $35-39,9$ & $\geq 40$ \\
\hline
\end{tabular}

Fonte: ABESO, 2016.

Tendo em vista as possíveis consequências da obesidade, como doenças cardiovasculares (DCV), diabetes mellitus tipo 2 (DM2), hipertensão, distúrbios osteomusculares e diversos tipos de câncer, são aplicadas diversas formas de tratamento: mudança de hábitos, incluindo uma dieta balanceada e com índice calórico adequado e a prática de atividades físicas, e os tratamentos medicamentosos e cirúrgicos (MORTAN et al., 2019; BRASIL, 2017).

Embora as alterações no estilo de vida sejam a primeira linha de terapia para a diminuição do peso, muitas vezes são insuficientes para atingir as metas necessárias e, nesses casos, são usados tratamentos cirúrgicos ou medicamentosos, podendo ser feita a associação entre os dois (GADDE et al., 2018).

O tratamento medicamentoso é feito com base em anorexígenos, sendo permitidos no Brasil quatro fármacos dessa classe: sibutramina, que inibe a recaptação de noradrenalina, serotonina (5-hidroxitriptamina, 5-HT) e dopamina, aumentando a sensação de saciedade e a termogênese; orlistate, um inibidor reversível das lipases pancreáticas e gástricas, que reduz a absorção de gordura do intestino; liraglutida, um agonista do peptídeo semelhante ao glucagon-1 (GLP-1), que provoca supressão do apetite e atraso no esvaziamento gástrico; e a lorcasserina, que ativa o receptor de serotonina 5HT2C no cérebro suprimindo, assim, o apetite (MORTANet al., 2019, BERSOUX et al., 2017).

A lorcasserina é o medicamento mais recente no Brasil, aprovada pela Agência Nacional de Vigilância Sanitária (ANVISA) em dezembro de 2016, mas só teve seus direitos concedidos para desenvolvimento e marketing no país em dezembro de 
2018, com o acordo feito entre Ensai e Eurofarma (ANVISA, 2016; Eisai, 2018). Segundo a classificação Anatomic Therapeutic chemical (ATC/DDD), o fármaco é um produto antiobesidade de ação central, tendo como código: A08AA11 (WHO, 2019).

O medicamento é indicado para perda de peso para pacientes que apresentam IMC igual ou superior a $30 \mathrm{~kg} / \mathrm{m}^{2}$ ou $27 \mathrm{~kg} / \mathrm{m} 2$ com a presença de ao menos uma comorbidade relacionada ao peso, como hipertensão arterial, apneia do sono e adoeças cardiovasculares. O fármaco possui dose recomendada de $10 \mathrm{mg}$ uma ou duas vezes ao dia e deve ser associado a uma dieta de redução de caloria e atividade física aumentada (BERSOUX et al., 2017).

As reações adversas a medicamentos (RAMs) mais comuns da lorcasserina são: dores de cabeça, nas costas e musculoesquelética, infecção do trato respiratório superior, nasofaringite, baixa quantidade de açúcar no sangue em pacientes com diabetes mellitus tipo 2.

Entre as RAMs mais graves estão: o aumento da prolactina, batimento cardíaco lento, alterações na memória e atenção, alucinações, depressão e diminuição na quantidade de células sanguíneas, priapismo e doença valvular cardíaca. Mulheres grávidas não devem tomar o medicamento (EUROFARMA, 2018).

Vale salientar que intervenções cirúrgicas e farmacológicas, necessitam da mudança de hábitos para a manutenção dos efeitos e para uma melhor ação e se existirem distúrbios neurológicos e endócrinos associados a obesidade, devem ser cuidados com tratamentosespecíficos.

O presente trabalho tem por objetivo averiguar a eficácia da lorcasserina na perda de peso em pessoas com IMC acima de $27 \mathrm{~kg} / \mathrm{m}^{2}$ e observar outros possíveis benefícios, como segurança cardiovascular e efeitos positivos em valores laboratoriais. 


\section{MÉTODO}

Foi realizada uma pesquisa bibliográfica exploratória, com abordagem integrativa, com uma síntese qualitativa dos trabalhos encontrados, o estudo foi realizado em dezembro de 2019 , sendo inclusos somente os artigos científicos publicados no período de janeiro de 2015 até a data de realização do presente estudo, tendo em vista que o fármaco está a pouco tempo no mercado, sendo relativamente um novo tratamento para a obesidade.

Foram utilizados os seguintes descritores lorcasserina; inibidores de apetite, perda de peso e obesidade, e seus equivalentes na língua inglesa. A pesquisa foi realizada com os descritores de maneira individualizada e dois a dois, sempre usando o termo "lorcasserina". A busca foi feita nas bases de dados eletrônicas: Scielo, Lilacs e Pubmed. A priori, foram encontradas respectivamente 4, 4, 84. Todos os 92 títulos e resumos foram analisados e foram eliminados 67 artigos por não indicaram em sua metodologia ter abordado a relação entre lorcasserina e a perda de peso.

Sendo assim, 25 artigos foram lidos na íntegra, nessa fase, foram eliminados outros 15 artigos por não atenderem ao objetivo esperado da pesquisa. Os 10 artigos restantes foram incluídos no estudo, dos quais foram destacados: ano de publicação, tipo de estudo realizado, objetivo e principais resultados obtidos.

\section{RESULTADOS E DISCUSSÕES}

Após a aplicação dos critérios de busca e seleção, foram encontrados 92 artigos, sendo destes 10 elegíveis. Na Tabela 1 estão apresentados os principais objetivos e resultados dos artigos selecionados para o presente estudo. 
Tabela 1: Principais Características dos Artigos Selecionados.

\begin{tabular}{|c|c|c|c|c|}
\hline Autores & $\begin{array}{c}\text { Revista e Ano } \\
\text { da } \\
\text { Publicação }\end{array}$ & $\begin{array}{l}\text { Tipo de } \\
\text { Estudo }\end{array}$ & Objetivo & Principais Resultados \\
\hline $\begin{array}{l}\text { FUJIOKA, K.; } \\
\text { PERDOMO, C.; } \\
\text { MALHOTRA, } \\
\text { M. }\end{array}$ & $\begin{array}{l}\text { Obesity } \\
\text { Science } \\
\text { Practice, } \\
2019 .\end{array}$ & $\begin{array}{l}\text { Análise } \\
\text { retrospectiva. }\end{array}$ & $\begin{array}{l}\text { Avaliar a perda } \\
\text { de peso com } \\
\text { lorcasserina em } \\
\text { pessoas com } \\
\text { apneia } \\
\text { obstrutiva do } \\
\text { sono (AOS). }\end{array}$ & $\begin{array}{l}\text { Houve redução significativa } \\
\text { de peso, comparada aos } \\
\text { pacientes que utilizaram } \\
\text { placebo, além da diminuição } \\
\text { da PA e da FC. Não houve } \\
\text { diferença entre pacientes } \\
\text { com ou sem AOS. }\end{array}$ \\
\hline ELHAG et al. & $\begin{array}{l}\text { Annals of } \\
\text { Medicine and } \\
\text { Surgery, } 2019 .\end{array}$ & $\begin{array}{l}\text { Revisão } \\
\text { retrospectiva } \\
\text { de registros. }\end{array}$ & 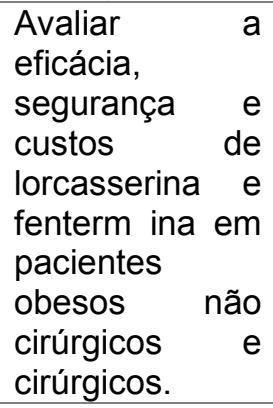 & $\begin{array}{l}\text { A fentermina foi mais eficaz } \\
\text { na redução do peso total e } \\
\text { do IMC, e possui um custo } \\
\text { menor, porém apresenta } \\
\text { mais efeitos colaterais que a } \\
\text { lorcasserina, nenhum dos } \\
\text { dois melhoraram a glicemia. }\end{array}$ \\
\hline FUJIOKA et al. & $\begin{array}{l}\text { Obesity } \\
\text { Science } \\
\text { Practice, } \\
2019 .\end{array}$ & $\begin{array}{l}\text { Análise post } \\
\text { hoc. }\end{array}$ & $\begin{array}{l}\text { Avaliar a perda } \\
\text { de peso com } \\
\text { lorcasserina em } \\
\text { adultos através } \\
\text { dos quartis de } \\
\text { idade. }\end{array}$ & $\begin{array}{l}\text { A lorcasserina se mostrou } \\
\text { eficaz na redução de peso } \\
\text { em todas as faixas etárias } \\
\text { analisadas, no entanto a } \\
\text { perda de peso demonstrou- } \\
\text { se ser maior para pacientes } \\
\text { mais velhos. }\end{array}$ \\
\hline BAYS et al. & $\begin{array}{l}\text { Science } \\
\text { Practice, } \\
2018 .\end{array}$ & $\begin{array}{l}\text { Análise } \\
\text { retrospectiva }\end{array}$ & $\begin{array}{l}\text { Quantificar a } \\
\text { contribuição } \\
\text { relativa da } \\
\text { perda de peso } \\
\text { para os efeitos } \\
\text { do tratamento } \\
\text { com } \\
\text { lorcasserina } 10 \\
\text { mg duas vezes } \\
\text { ao dia nos } \\
\text { principais } \\
\text { parâmetros } \\
\text { metabólicos. }\end{array}$ & $\begin{array}{lr}\text { A lorcasserina } & 10 \mathrm{mg} \text { BID } \\
\text { melhorou } & \text { diversas } \\
\text { anormalidades } & \text { metabólicas } \\
\text { adiposopáticas } & \text { com } \\
\text { contribuições } & \text { variáveis } \\
\text { atribuíveis à perda de peso, } \\
\text { como PA e circunferência da } \\
\text { cintura. }\end{array}$ \\
\hline $\begin{array}{l}\text { HURT, R.T.; } \\
\text { MUNDI, M. S.; } \\
\text { EBBERT, J.O. }\end{array}$ & $\begin{array}{l}\text { Metabolic } \\
\text { Syndrome and } \\
\text { Obesity: } \\
\text { Targets and } \\
\text { Therapy, } 2018\end{array}$ & $\begin{array}{l}\text { Revisão } \\
\text { bibliográfica. }\end{array}$ & $\begin{array}{l}\text { Avaliar os } \\
\text { ensaios clínicos } \\
\text { envolvendo a } \\
\text { liberação } \\
\text { imediata (IR) do } \\
\text { lorcasserina e o } \\
\text { lorcasserina XR } \\
\text { nas áreas de } \\
\text { obesidade, } \\
\text { diabetes } \\
\text { dependência. }\end{array}$ & $\begin{array}{llr}\text { O lorcasserina } & \text { IR } \\
\text { demonstrou eficácia na } \\
\text { perda de } & \text { peso } \\
\text { bioequivalência com a } \\
\text { lorcasserina XR, além de } \\
\text { auxiliar no controle do } \\
\text { tabagismo e possivelmente } \\
\text { da glicemia. }\end{array}$ \\
\hline CHAO et al. & $\begin{array}{l}\text { Cravings, and } \\
\text { Appetite. Clin } \\
\text { Obes, } 2018 .\end{array}$ & $\begin{array}{l}\text { Ensaio } \\
\text { clínico } \\
\text { randomizado. }\end{array}$ & $\begin{array}{lr}\text { Avaliar } & \text { os } \\
\text { efeitos } & \text { da } \\
\text { lorcasserina } & \text { em }\end{array}$ & $\begin{array}{l}\text { Houve melhora semelhante } \\
\text { na alimentação relacionada à } \\
\text { emoção e ao estresse no }\end{array}$ \\
\hline
\end{tabular}


BOHULA et al. N Engl J Med, 2018.
24 semanas uso da lorcasserina e da após a dieta com baixa caloria. No randomização entanto, o uso dos dois na alimentação combinados mostrou relacionada à maiores benefícios em emoção e ao relação ao placebo. estresse.

Avaliar segurança Em uma população de alto a risco de pacientes com cardiovascular lorcasserina facilitou a perda do uso da sustentada de peso e baixou lorcasserina em ligeiramente fatores de risco pacientes com sobrepeso ou obesidade. cardiovasculares e metabólicos em relação ao placebo.

A fentermina em conjunto com a lorcasserina aumentou a perda de peso a Avaliar a curto prazo, mas não tolerabilidade a aumentou a incidência de curto prazo da EAs potencialmente $\begin{array}{llll} & \text { Obesity (Silver } & \text { Ensaio } & \text { curto prazo da } \\ \text { SMITH et al. } & \text { Spring, } & \text { clínico } & \text { lorcasserina } \\ & \text { Maryland), } & \text { randomizado. } & \begin{array}{l}\text { isolada ou com } \\ \text { dois regimes }\end{array} \\ & 2017 & & \begin{array}{l}\text { posológicos de } \\ \text { fentermina. }\end{array}\end{array}$ serotoninérgicos, no entanto, a fentermina duas vezes ao dia aumentou a descontinuação em comparação com a lorcasserina isolada e a lorcasserina mais a fentermina uma vez aodia.

\begin{tabular}{|c|c|c|c|c|}
\hline FARR et al. & $\begin{array}{l}\text { Diabetes, } \\
2016\end{array}$ & $\begin{array}{l}\text { Ensaio } \\
\text { clínico } \\
\text { randomizado. }\end{array}$ & $\begin{array}{lr}\text { Examinar os } \\
\text { possíveis alvos } \\
\text { do CNS para a } \\
\text { lorcasserina } \\
\text { usando(fMRI) e } \\
\text { estudar como a } \\
\text { lorcasserina } \\
\text { altera } & \text { as } \\
\text { ativações } & \text { do } \\
\text { centro } & \text { do } \\
\text { cérebro } & \text { em } \\
\text { resposta } & \text { a } \\
\text { imagens } & \text { de } \\
\text { alimentos a } & \text { a } \\
\text { curto prazo } & (1 \\
\text { semana) } & \text { e } \\
\text { longo prazo } & (4 \\
\text { semanas). } & \end{array}$ & $\begin{array}{l}\text { A lorcasserina exerce seus } \\
\text { efeitos de redução de peso, } \\
\text { diminuindo as ativações } \\
\text { cerebrais relacionadas à } \\
\text { atenção a sinais de comida } \\
\text { (córtices parietais e visuais) } \\
\text { e atividade emocional e } \\
\text { límbica (ínsula, amígdala). }\end{array}$ \\
\hline $\begin{array}{l}\text { GUTIERREZ et } \\
\text { al. }\end{array}$ & $\begin{array}{l}\text { Hepatology, } \\
2016\end{array}$ & $\begin{array}{l}\text { Estudo } \\
\text { caso. }\end{array}$ & $\begin{array}{ll}\text { Avaliar } & \text { os } \\
\text { efeitos } & \text { da } \\
\text { lorcasserina na } & \text { diminuição de } \\
\text { peso de uma } \\
\text { paciente pré/- } \\
\text { transplante de } \\
\text { fígado. }\end{array}$ & $\begin{array}{l}\text { O uso da lorcasserina } \\
\text { auxiliou na perda de peso e } \\
\text { na melhora do IMC e de } \\
\text { diversos valores } \\
\text { laboratoriais. O medicamento } \\
\text { pode ser usado como ponte } \\
\text { para cirurgia bariátrica ou } \\
\text { transplante de fígado. }\end{array}$ \\
\hline
\end{tabular}


Foram analisados 10 estudos sobre a perda de peso com o uso da lorcasserina em pacientes com obesidade ou sobrepeso, com pelo menos uma comorbidade relacionada a doença, sendo na maioria o fármaco associado a prática de atividade física e alimentação adequada. Os trabalhos trouxeram análises sobre pacientes cirúrgicos e não-cirúrgicos e com algumas patologias como: apneia do sono e diabetes mellitus tipo 2.

A lorcasserina apresentou eficácia em todos os estudos analisados, como descrevem Fujikoka et al. (2019) e Bohula et al. (2018), onde cerca de 47\% dos pacientes tiveram redução de pelo menos $5 \%$ do peso corporal e $22 \%$, reduziram $10 \%$ ou mais. Os resultados foram bem melhores dos que os dos pacientes que utilizaram apenas o placebo, no qual apenas $25,6 \%$ tiveram redução de pelo menos $5 \%$ do peso e $13 \%$ de mais de $10 \%$.

Acreditasse que a redução de peso seja devido a diminuição do apetite causado pela melanocortina 4 (MC4). Esse fármaco é altamente seletivo para os receptores centrais $5-\mathrm{HT} 2 \mathrm{C}$, que são receptores acoplados a proteína $\mathrm{G}$ presente no plexo coroide cerebral, no córtex cerebral, nos gânglios da base e no sistema límbico. A lorcasserina provavelmente atua sobre a área do núcleo arqueado do hipotálamo, através de áreas neurais com funções de balanço energético e ativa os neurônios pró-opiomielanocortina (POMC), resultando em sinalização de segunda ordem via receptores de 4 MC4 (BAYS et al., 2018).

A perda do apetite acontece também em casos de fome causada por questões emocionais, estresse, compulsão e ansiedade (CHAO et al., 2018).

Pensa-se que, além da redução da fome a lorcasserina causa outros benefícios cerebrais, como a melhora cognitiva e a redução de dependências químicas. Em alguns estudos feitos em camundongos houve a diminuição do vício por álcool, nicotina e cocaína (FARR et al., 2016; HURT; MUNDI; EBBERT, 2018).

Além de auxiliar na reversão da obesidade, a lorcasserina auxiliou em outras patologias, geralmente associadas ao excedente de peso, pacientes com diabetes mellitus tipo dois apresentaram melhora na tolerância a insulina e nos níveis glicêmicos sanguíneos, devido ao efeito da serotonina ou o 5 - HT, um neurotransmissor que medeia a homeostase da glicose. Também houve melhora em 
pessoas com apneia obstrutiva do sono e artrite (BAYS et al., 2018; HURT; MUNDI; EBBERT, 2018; FUJIOKA; PERDOMO; MALHOTRA, 2019).

Ademais, houve melhora de diversos fatores laboratoriais, como redução da pressão arterial, da frequência cardíaca e do colesterol, que diminuem os riscos de doenças cardíacas, também não foi apresentado aumento no número de eventos cardíacos (composto de morte cardiovascular, infarto do miocárdio ou acidente vascular cerebral) maior que nos pacientes que utilizaram placebo (GUTIERREZ et al., 2016; HURT; MUNDI; EBBERT, 2018; FUJIOKA etal., 2019).

Outro fator que distingue a lorcasserina da maioria dos outros anorexígenos é a baixa evidencia de efeitos adversos: 9,9\% dos pacientes apresentaram efeitos adversos comparado a $18 \%$ da fentermina, por exemplo, além disso, os efeitos geralmente são mais brandos como dor de cabeça ou tontura (ELHAG et al., 219; SMITH et al., 2017).

\section{CONCLUSÃO}

A obesidade é um dos maiores problemas de saúde pública dos últimos anos e para a prevenção e tratamento dessa doença é importante uma adequação na quantidade de calorias consumidas e na prática de exercícios físicos, no entanto, nem sempre isso é o suficiente quandoas modificações no estilo de vida não resultam na perda de peso desejada, a farmacoterapia é uma opção bastante viável.

A lorcasserina, que é o medicamento mais recentemente liberado no Brasil, apresenta efeitos positivos quanto a redução de peso, tendo porcentagens de redução de IMC semelhantesa outros fármacos, além de auxiliar no controle da fome relacionada a questões emocionais, como ansiedade.

O fármaco apresentou, ainda, vantagem em relação aos outros anorexígenos devido a sua maior segurança cardiovascular, não foi evidenciado eventos cardiovasculares adversos maiores e ocasionou melhoria em vários fatores de risco como: hipertensão arterial, lipidemia e glicemia elevados e disfunção renal. A lorcaserina também apresentou baixa incidência de efeitos adversos. 


\section{REFERÊNCIAS BIBLIOGRÁFICAS}

ASSOCIAÇÃO BRASILEIRA PARA O ESTUDO DA OBESIDADE E DA SÍNDROME METABÓLICA. Diretrizes brasileiras de obesidade. 4. ed. São Paulo: ABESO, 2016. Disponível em: <http://www.abeso.org.br/uploads/downloads/92/57fccc403e5da.pdf>. Acesso em: dez. de 2019.

BAYS, H.; PERDOMO, C.; NIKONOVA, E.; KNOT, R.; MALHOTRA, M. Lorcaserin and metabolic disease: weight-loss dependent and independent effects. Obesity Science \& Practice, vol.: 4, pág.: 499-505, 2018.

$B E L V I Q \circledR$ : cloridrato de lorcaserina. Responsável técnico: Luiz Rogério M. Silva. São Paulo SP: Eurofarma Laboratórios S.A., 2018. Bula de remédio.

BERSOUX, S.; BYUN, T. H.; SWARNA, S. C.; POOLE, JR, K. G. Pharmacotherapy for obesity: What you need to know. Cleveland Clinic Journal of Medicine. 84(12):951-958, Ohio, dez. de 2017.

BOHULA, E. A.; WIVIOTT, S. D.; MCGUIRE, D. K. INZUCCHI, S. E.; KUDER, J.; FANOLA, C. L. et al. Cardiovascular Safety of Lorcaserin in Overweight or Obese Patients. N Engl J Med, vol.: 379, pág.: 1107-1117, 2018.

BRASIL, Agência Nacional de Saúde Suplementar. Manual de diretrizes para o enfretamentoda obesidade na saúde suplementar brasileira. Agência Nacional de Saúde Suplementar. Diretoria de Normas e Habilitação dos Produtos. Gerência-Geral de Regulação Assistencial.Gerência de Monitoramento Assistencial. Coordenadoria de Informações Assistenciais. Rio de Janeiro: Anvisa, 2017.

BRASIL, Ministério da saúde. Vigitel Brasil 2018: vigilância de fatores de risco e proteçãopara doenças crônicas por inquérito telefônico: estimativas sobre frequência e distribuição sociodemográfica de fatores de risco e proteção para doenças crônicas nas capitais dos 26 estados brasileiros e no Distrito Federal em 2018 / Ministério da Saúde, Secretaria de Vigilância em Saúde, Departamento de Análise em Saúde e Vigilância de Doenças não Transmissíveis. Brasília: Ministério da Saúde, 2019.

BRASIL. Ministério da Saúde. Agência Nacional de Vigilância Sanitária. ANVISA libera novo insumo farmacêutico para obesidade. 2017. Disponível em: $<$ http://portal.ANVISA.gov.br/noticias/-/asset_publisher/FXrpx9qY7FbU/content/ANVISA- liberanovo-insumo-farmaceutico-paraobesidade/219201/pop up? 101 INSTANCE FXrpx9qY7FbU viewMode=print\& 101 INS TANCE_FXrpx9qY7FbU_languageld=pt_BR>. Acesso em: dez. de 2019.

CARVALHO, A.R.M. BELÉM, M.O. ODA, J.Y. Sobrepeso e obesidade em alunos de 6-10anos de escola Estadual de Umuarama/PR. Arq Ciências Saúde UNIPAR, vol: 21, pág.: 3-12, 2017.

CHAO, A.M. et al. A Randomized Controlled Trial of Lorcaserin and Lifestyle Counseling for Weight Loss Maintenance: Changes in Emotion- and Stress-Related Eating, Food Cravings, and Appetite. Clin Obes, vol.: 8, pág.: 383-390, 2018.

DIAS, P.C. HENRIQUES, P. ANJOS, L. A. BURLANDY, L. Obesidade e políticas públicas: concepções e estratégias adotadas pelo governo brasileiro. Cad. Saúde Pública 33 (7) 27 jul. 2017.

\section{EISAI SIGNS COLLABORATION AGREEMENT FOR ANTI-OBESITY AGENT LORCASERIN IN}


BRAZIL WITH EUROFARMA. Eisai Co., Ltd. No.18-106, 2018.

ELHAG, W. et al. Lorcaserin vs. Phentermine among non-surgical and surgical obese patients: Anthropometric, glycemic, lipid, safety and cost outcomes. Annals of Medicine and Surgery, vol.: 45, p.: 75-8, 2019.

FARR, O. M. UPADHYAY, J.; GAVRIELI, A.; CAMPO, M.; SPYROU, N.; KAYE, H. et al. Lorcaserin Administration Decreases Activation of Brain Centers in Response to Food Cues and These Emotion- and Salience-Related Changes Correlate With Weight Loss Effects: A 4-WeekLong Randomized, Placebo-Controlled, Double-Blind Clinical Trial. Diabetes vol. 65 (10), p. 2943-2953, 2016.

FUJIOKA, K.; MALHOTRA, M.; PERDOMO, C.; APOVIAN, C. M. Effect of lorcaserin in different age groups: a post hoc analysis of patients from the BLOOM, BLOSSOM and BLOOM-DM studies. Obesity Science \& Practice, vol.: 5, p.: 120-129, 2019.

FUJIOKA, K.; PERDOMO, C.; MALHOTRA, M. Effect of lorcaserin on weight reduction in persons with obstructive sleep apnea (OSA): a combined subgroup analysis from three randomized, controlled clinical trials. Obesity Science \& Practice, vol.: 5, p.: 238-245, 2019.

GADDE, K. M.; MARTIN, C. K.; BERTHOUD, H.R.; HEYMSFIELD, S. B. Obesidade: Fisiopatologia e Manejo. American College of Cardiology Foundation, 71 (1): 69-84, Nova Jersey. Jan. de 2018.

GUTIERREZ, J.A.; LANDAVERDE, C.; WELLS, J.T.; POOTDAD, F. Lorcaserin Use in the Management of Morbid Obesity in a Pre-Liver Transplant Patient. Hepatology, vol. 64, p.:301302, 2016.

HURT, R.T.; MUNDI, M. S.; EBBERT, J.O. Challenging obesity, diabetes, and addiction:the potential of lorcaserin extended release. Metabolic Syndrome and Obesity: Targets and Therapy, vol.: 11 p.: 469-478, set. de 2018.

Instituto Brasileiro de Geografia e Estatística. Pesquisa Nacional de Saúde 2013: ciclos de vida: Brasil e grandes regiões. Rio de Janeiro: Instituto Brasileiro de Geografia e Estatística; 2015.

MELO, M. E. PINHO, A. C. Câncer e obesidade: Um alerta do INCA. Rede Câncer, edição38, p.: 34-35. ago. 2017.

MORTAN, P.D. SOURLAS, A.; OLIVERO, J.; SILVERIO, D.; GUZMAN, E.; KOSMAS, C. E. Pharmacologic therapy of obesity: mechanisms of action and cardiometabolic effects. Annals of Translational Medicine Vols. 7 (16). Ago. de 2019.

SMITH, S. R. GARVEV, W. T., GREENWAY, F.L.; ZHOU, S.; FAIN, R.; PILSON, R. et al. Coadministration of lorcaserin and phentermine for weight management: $A$ 12-week, randomized, pilot safety study. Obesity (Silver Spring, Maryland), vol. 25, p. 857-865, 2017.

SOUZA, S. A.; SILVA, A. B.; CAVALCANTE, U. M. B.; LIMA, C. M. B. L.; SOUZA, T. C. Obesidade adulta nas nações: uma análise via modelos de regressão beta. Cad. Saúde Pública 34 (8). 20 ago. 2018. 